\title{
Sirt1 regulates canonical TGF- $\beta$ signalling to control fibroblast activation and tissue fibrosis
}

\author{
Pawel Zerr, ${ }^{1}$ Katrin Palumbo-Zerr, ${ }^{1}$ Jingang Huang, ${ }^{1}$ Michal Tomcik, ${ }^{1,2}$ \\ Barbora Sumova, ${ }^{1,2}$ Oliver Distler, ${ }^{3}$ Georg Schett, ${ }^{1}$ Jörg H W Distler ${ }^{1}$
}

\begin{abstract}
Handling editor Tore K Kvien
- Additional material is

published online only. To view please visit the journal online (http://dx.doi.org/10.1136/ annrheumdis-2014-205740).

${ }^{1}$ Department of Internal Medicine III, Institute for Clinical Immunology, University of Erlangen-Nuremberg, Erlangen, Germany

${ }^{2}$ Department of Rheumatology, 1st Faculty of Medicine, Institute of Rheumatology, Charles University, Prague,

Czech Republic

${ }^{3}$ Center of Experimental Rheumatology and Zurich Center of Integrative Human Physiology, University Hospital Zurich, Zurich, Switzerland
\end{abstract}

\section{Correspondence to} Dr Jörg H W Distler, Department of Internal Medicine 3, Institute for Clinical Immunology, University of Erlangen-Nuremberg, Ulmenweg 18, 91054 Erlangen, Germany; joerg.distler@uk-erlangen.de

Received 14 April 2014 Revised 4 August 2014 Accepted 10 August 2014 Published Online First 1 September 2014

\section{ABSTRACT}

Background Sirt1 is a member of the sirtuin family of proteins. Sirt1 is a class III histone deacetylase with important regulatory roles in transcription, cellular differentiation, proliferation and metabolism. As aberrant epigenetic modifications have been linked to the pathogenesis of systemic sclerosis (SSC), we aimed to investigate the role of Sirt1 in fibroblast activation. Methods Sirt1 expression was analysed by real-time PCR, western blot and immunohistochemistry. Sirt1 signalling was modulated with the Sirt1 agonist resveratrol and by fibroblast-specific knockout. The role of Sirt1 was evaluated in bleomycin-induced skin fibrosis and in mice overexpressing a constitutively active transforming growth factor- $\beta$ (TGF- $\beta$ ) receptor I (TBRlact).

Results The expression of Sirt1 was decreased in patients with SSC and in experimental fibrosis in a TGF$\beta$-dependent manner. Activation of Sirt1 potentiated the profibrotic effects of TGF- $\beta$ with increased Smad reporter activity, elevated transcription of TGF- $\beta$ target genes and enhanced release of collagen. In contrast, knockdown of Sirt1 inhibited TGF- $\beta / S M A D$ signalling and reduced release of collagen in fibroblasts. Consistently, mice with fibroblast-specific knockdown of Sirt1 were less susceptible to bleomycin- or TBRlact-induced fibrosis.

Conclusions We identified Sirt1 as a crucial regulator of TGF- $\beta /$ Smad signalling in SSC. Although Sirt1 is downregulated, this decrease is not sufficient to counterbalance the excessive activation of TGF- $\beta$ signalling in SSc. However, augmentation of this endogenous regulatory mechanism, for example, by knockdown of Sirt1, can effectively inhibit TGF- $\beta$ signalling and exerts potent antifibrotic effects. Sirt1 may thus be a key regulator of fibroblast activation in SSc.

\section{INTRODUCTION}

Systemic sclerosis (SSc) is a chronic fibrotic connective disease of unknown aetiology that affects the skin and internal organs. SSc is characterised by a persistent mesenchymal tissue response with aberrant activation of fibroblasts, resulting in excessive release of extracellular matrix. ${ }^{1}$ The accumulating of extracellular matrix proteins disturbs the physiological tissue function and causes high morbidity and mortality. ${ }^{2}$ Transforming growth factor- $\beta$ (TGF- $\beta$ ) has been characterised as a key mediator of fibroblast activation in SSc and other fibrotic diseases. ${ }^{3}$ However, the intracellular signalling cascades that control TGF- $\beta$ signalling and the TGF- $\beta$-induced activation of fibroblasts are still incompletely understood.
Sirtuins are a class of proteins that gained major interest due to potential implications in longevity. Sirtuins have been implicated in a wide range of cellular processes like transcription, apoptosis, stress resistance and metabolism. Furthermore, sirtuins also regulate circadian rhythm, mitochondrial biogenesis and inflammation. ${ }^{4}$ While only one sirtuin is found in lower eukaryotes (Sir2: Silent Information Regulator 2), mammals encode for seven different sirtuins, Sirt1-7, that occupy different subcellular compartments such as the nucleus (Sirt1, -2, -6, -7), cytoplasm (Sirt1 and Sirt2) and the mitochondria (Sirt3, -4 and -5). Among the different members of the sirtuin family, Sirt 1 is the most studied and the most closely related to Sir 2 in eukaryotes. ${ }^{5}$ Sirt1 is an NAD-dependent deacetylase with a broad range of cellular substrates. Histones are a major target of Sirt1, and Sirt1 regulates the transcription of a plethora of genes indirectly by histone-dependent epigenetic silencing. Indeed, Sirt1 is categorised as a class III histone deacetylase. ${ }^{6}$ However, in addition to deacetylation of histones, numerous non-histone targets of Sirt1 have been identified more recently. These nonhistone substrates of Sirt1 also include a variety of transcription factors. ${ }^{7}$ Of particular interest for SSc, Sirt1 has recently been demonstrated to deacetylate Smad3 and Smad7, thereby promoting ubiquitination and subsequent degradation of these proteins. ${ }^{8} 9$ Moreover, Sirt1 has been shown to regulate endogenous responses to oxidative stress, which is considered to play a key role in the pathogenesis of SSc. ${ }^{1}$ Sirt1 can stimulate the expression of antioxidants via FoxO-dependent pathways. ${ }^{10} 11$ On the other hand, increased levels of reactive oxygen species (ROS) can inhibit Sirt1 activity by evoking oxidative modifications on its cysteine residues. ${ }^{12}$ Finally, Sirt1 has been shown to inhibit the expression of matrix-metallo-proteinases (MMP) 1 and $3 .{ }^{13}$ Considering the effects of Sirt 1 on Smad proteins, its role in oxidative stress responses and for the regulation of MMP expression, we hypothesised that Sirt1 might play a role in the aberrant fibroblast activation in SSc.

\section{MATERIALS AND METHODS}

\section{Patients and fibroblast cultures}

Skin biopsies of 17 patients with SSc and 15 healthy volunteers matched for age and sex. All patients with SSc fulfilled the 1980 American College of Rheumatology (ACR) criteria as well as the 2013 ACR/ European League Against Rheumatism Response (EULAR) criteria for SSc. ${ }^{14}$ 
In total, 12 patients had diffuse cutaneous SSc, 5 limited cutaneous SSc, 12 were women and 5 were men. The median age of patients with SSc was 50 years (range 30-71 years) and their median disease duration was 5 years (range $0.5-12$ years). Eight patients had active disease as defined by the EULAR Scleroderma Trials and Research (EUSTAR) activity score. All biopsies were taken from involved skin at the forearm. Patients with SSc and healthy volunteers signed a consent form approved by the local institutional review boards.

For the in vitro experiments, fibroblasts were obtained from patients with SSc, healthy volunteers, mice conditional deficient for Sirt 1 and wild-type littermates used at passages from 4-8 as previously described. ${ }^{15}{ }^{16}$ In selected experiments, fibroblasts were stimulated with combinations of TGF- $\beta$ at a concentration of $10 \mathrm{ng} / \mathrm{mL} \quad(\mathrm{R} \& \mathrm{D}$ Systems, Wiesbaden-Nordenstadt, Germany) and/or resveratrol at a concentration of $50 \mu \mathrm{M}$ (Sigma-Aldrich, Wiesbaden, Germany).

\section{Immunofluorescence staining}

Immunofluorescence staining for Sirt1 was performed according to previously established protocols. ${ }^{17}{ }^{18}$ Human and murine skin sections and corresponding controls were incubated with the primary polyclonal rabbit anti-Sirt1 antibodies (Abcam, Cambridge, UK) and visualised with red fluorescence dye Alexa Fluor 594 as secondary antibodies (Life Technologies, Darmstadt, Germany). Polyclonal mouse anti-Vimentin (Abcam) was used for counter staining, visualised with a green fluorescence dye Alexa Fluor 488 as secondary antibodies (Life Technologies). Cell nuclei were stained with 4',6-diamidino2-phenylindole (DAPI). Images were captured at a 200-fold and a 1000 -fold magnification where indicated.

\section{Western blot analysis}

Protein samples transferred to polyvinylidene fluoride membranes were incubated with polyclonal rabbit anti-Sirt1 antibodies (Abcam) in a dilution of $1: 100$ in $2 \%$ bovine serum albumin/phosphate buffered saline. $\beta$-Actin served as a loading control. Species-specific IgGs labelled with horseradish peroxidase (Dako, Hamburg, Germany) served as secondary antibodies. The ECL Plus Western Blotting Detection System and highperformance chemiluminescence films were used for visualisation.

\section{Quantitative real-time PCR}

Gene expression was quantified by SYBR Green real-time PCR using the MxPro-3500P Sequence Detection System (Agilent, Böblingen, Germany) as previously described. ${ }^{18}{ }^{19}$ All primer sequences are shown in online supplementary table S1. False positive signals caused by primer dimers were excluded by nontemplate controls and by dissociation curve analysis. The amounts of cDNA were normalised by $\beta$-actin housekeeping gene.

\section{Quantification of collagen protein}

The amount of soluble collagen in cell culture supernatants was quantified using the SirCol collagen assay (Biocolor, Belfast, Northern Ireland) as previously described. ${ }^{20} 21$

\section{Analysis of myofibroblast differentiation}

To evaluate the effects of Sirt1 on myofibroblast differentiation, the formation of stress fibres and the expression levels and pattern of $\alpha$-smooth muscle actin ( $\alpha S M A)$ protein were analysed in dermal fibroblasts cultured in chamber slides. Stress fibres were visualised with phalloidin as described previously. ${ }^{18} 2223$ $\alpha$ SMA expression was visualised via immunofluorescence staining with primary anti- $\alpha \mathrm{SMA}$ antibodies and secondary green fluorescence dye Alexa Fluor 488 antibodies (Life Technologies). Nuclei were counterstained with DAPI (Santa Cruz Biotechnology, Heidelberg, Germany).

\section{SMAD reporter assays}

Human dermal fibroblasts were transfected with replicationdeficient adeno-associated viruses encoding for luciferase under the control of a CAGA/Smad binding site (AdCAGA-Luc) at an infectious unit (IFU) of 800 . The effects of TGF- $\beta(10 \mathrm{ng} / \mathrm{mL})$ and resveratrol on the CAGA/Smad reporter were analysed by measuring the luciferase activity in a microplate luminometer (Berthold Technologies, Germany). ${ }^{20} 24$

\section{Mouse models of fibrosis}

Two different mouse models were employed: bleomycininduced skin fibrosis and fibrosis induced by fibroblast-specific overexpression of a constitutively active TGF- $\beta$ receptor I (TBRIact). ${ }^{25}$

For bleomycin-induced skin fibrosis, 6-week-old mice received subcutaneous injections of bleomycin every other day for 4 weeks in defined areas of the upper back. ${ }^{26-28}$ Mice injected with equal volumes of $0.9 \%$ sodium chloride served as controls.

For TBRIact-induced dermal fibrosis, Col1a2 CreER mice were crossbred with TBRIact mice to generate double transgenic Col1a2 CreER; TBRIact that express TBRIact selectively in fibroblasts upon activation of Cre recombinase by tamoxifen. Col1a2 CreER; TBRIact mice were injected with tamoxifen at an age of 6 weeks (referred to as $\operatorname{Sirt} 1^{\mathrm{f} / \mathrm{fl}}$; Fib Cre ON mice). Injections of corn oil served as controls (Sirt $1^{\mathrm{fl} / \mathrm{fl}}$; Fib Cre OFF mice). The outcome was evaluated after 5 months. ${ }^{29}$

\section{Modulation of Sirt1 activity}

For inactivation of Sirt1, a Cre-LoxP-based approach was chosen. Sirt1 was selectively depleted in fibroblasts by crossbreeding mice expressing conditional alleles of Sirt1 (Sirt $1^{\mathrm{fl} / \mathrm{fl}}$ ) (Jackson Laboratory, Bar Harbor, Maine, USA) with Col1a2CreER mice. Sirt $1{ }^{\mathrm{f} / \mathrm{fl}}$; Col1a2CreER mice were injected with tamoxifen to induce Cre-mediated recombination and fibroblast-specific depletion of Sirt1.

\section{Histological analysis}

Skin sections were stained by haematoxylin and eosin. Dermal thickness was analysed with a Nikon Eclipse 80i microscope (Nikon, Badhoevedorp, Netherlands) at 100-fold magnification by measuring the distance between the epidermal-dermal junction and the dermal-subcutaneous fat junction at sites of induration at three consecutive skin sections of each animal. ${ }^{30-33}$

\section{Hydroxyproline assay}

Total collagen protein in skin samples was determined via hydroxyproline assay and quantified by Spectra MAX 190 microplate spectrophotometer as previously described. ${ }^{34-37}$

\section{Quantification of myofibroblasts}

Skin sections were stained with anti- $\alpha$ SMA (Sigma-Aldrich, Steinheim, Germany) antibodies. Secondary species-specific immunoglobulins labelled with horseradish peroxidase (Dako, Glostrup, Denmark) were used to visualise $\alpha$ SMA-positive myofibroblasts with a 3,3'-diaminobenzidine peroxidase substrate solution (Sigma-Aldrich). $\alpha$ SMA-stained sections were counterstained with haematoxylin. The number of myofibroblasts was 
determined at 200-fold magnification. Counting was performed in a blinded manner by two independent examiners. ${ }^{38-40}$

\section{Statistics}

All data are presented as median with IQR, and differences between the groups were tested for their statistical significance by non-parametric Mann-Whitney U test. p Values $<0.05$ were considered significant.

\section{RESULTS}

The expression of Sirt1 is downregulated in fibrotic skin

We first analysed the mRNA levels of Sirt1 in the skin of SSc and observed a significant decrease of Sirt1 mRNA compared with matched healthy individuals (figure 1A). Immunohistochemistry confirmed the reduced expression of Sirt1 in the skin sections of patients with SSc with prominently decreased levels of Sirt1 in spindle-shaped cells in the dermis (figure 1B). Double staining with the mesenchymal marker vimentin indicates that SSc fibroblasts express only low levels of Sirt1, whereas fibroblasts in healthy skin intensely stain for Sirt1 (figure 1B). No differences in the expression levels of Sirt1 were noted between patients with limited cutaneous SSc and diffuse cutaneous SSc. Although there was a trend towards lower levels of Sirt1 in patients with SSc with active disease, this did not reach statistical significance. Consistent with the findings in SSc, Sirt1 expression was also decreased in bleomycin-induced skin fibrosis with reduced mRNA and protein levels in skin lysates (figure 1C, D) and low levels of Sirt1 in fibroblasts ex vivo (figure 1E).

\section{Persistent activation of TGF- $\beta$ signalling downregulates the expression of Sirt1}

As persistent activation of TGF- $\beta$ signalling is a hallmark of SSc, we investigated whether TGF- $\beta$ signalling may regulate the expression of Sirt1. Indeed, exposure to chronically elevated levels of TGF- $\beta$ significantly decreased the mRNA and protein levels of Sirt1 in cultured human dermal fibroblasts (figures 2A, B). Overexpression of TBRIact also reduced the mRNA levels of Sirt1 in murine skin (figure 2C) and decreased Sirt1 expression in vimentin-positive dermal fibroblasts (figure 2D).

\section{Activation of Sirt1 stimulates TGF- $\beta$-induced fibroblast activation}

As feedback regulation is common within TGF- $\beta$ cascades, we next analysed whether Sirt1 might in turn modulate TGF- $\beta$-induced fibroblast activation. Activation of Sirt with

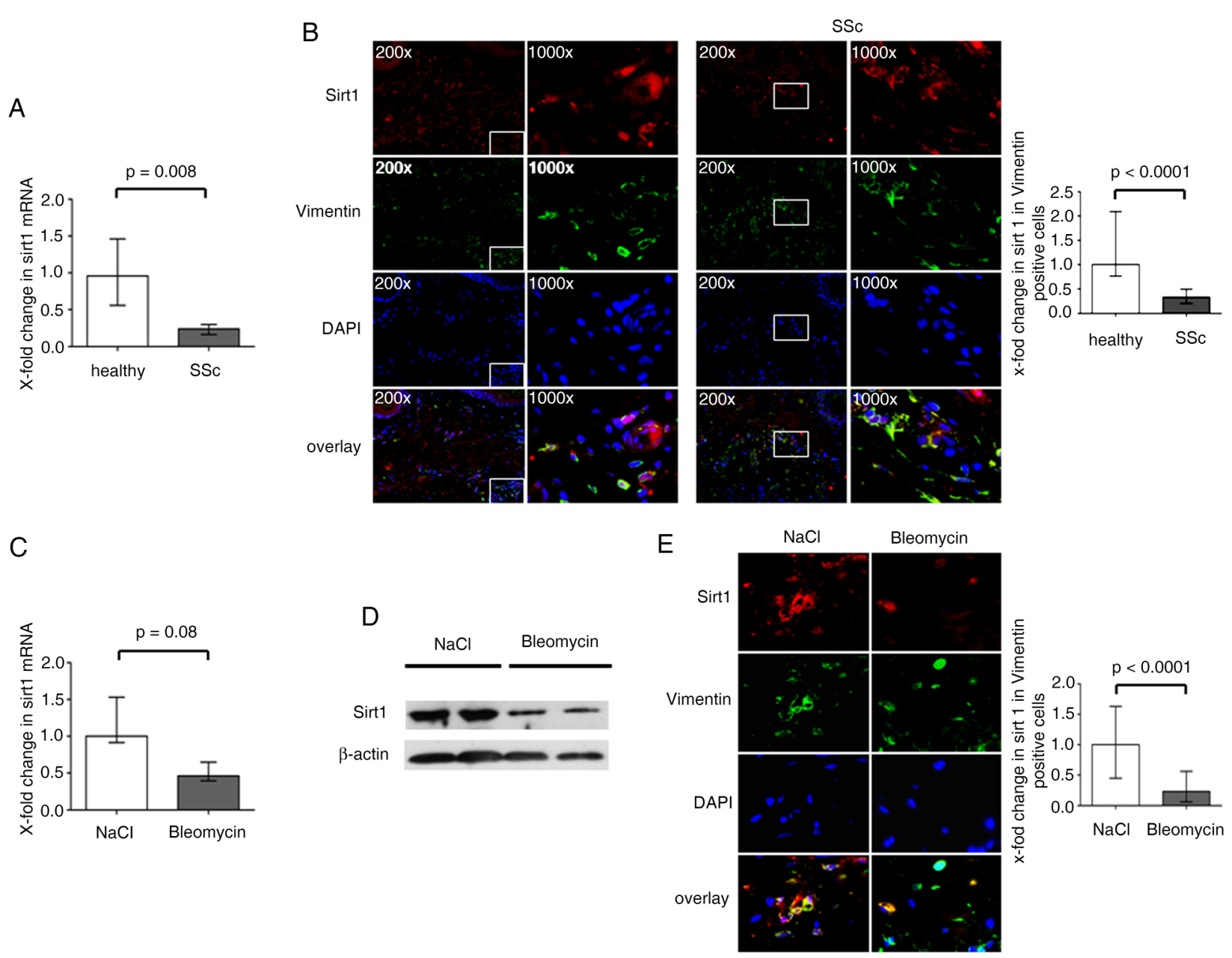

Figure 1 Sirt1 expression is decreased in SSc and in experimental fibrosis. (A, B) Sirt1 in SSc skin. (A) The mRNA levels of Sirt1 in SSc skin and normal skin analysed by qPCR $(n=6)$. (B) Sirt1 expression in vimentin-positive cells in the skin of patients with SSC and healthy volunteers shown by immunofluorescence ( $n=6$ for healthy and $n=6$ for SSC). (C-E) Levels of Sirt1 in bleomycin-induced fibrosis analysed by $q P C R(C)$ ( $n=6$ for each group), western blot (D) ( $n=6$ for each group) and immunofluorescence staining ( $E$ ) ( $n=6$ for each group). Representative immunofluorescence stainings are shown at a 200 -fold and 1000 -fold magnification, respectively. All data are presented as median with IQR. SSc, systemic Sclerosis; DAPI, 4',6-diamidino-2-phenylindole; qPCR, quantitative real-time PCR. 
Figure 2 Sirt1 is reduced in a TGF- $\beta$-dependent manner. mRNA (A) $(n=5$ for each condition) and protein (B) $(n=4$ for each condition) levels of Sirt1 upon chronic stimulation with TGF- $\beta$ every $12 \mathrm{~h}$ as determined by quantitative real-time $P C R$ and western blot. Sirt1 mRNA (C) ( $n=6$ for each group of mice) and protein (D) $(n=5$ for each group) levels in the skin of mice overexpressing TBRlact. All data are presented as median with IQR. TGF- $\beta$, transforming growth factor $\beta$; TBRlact, active transforming growth factor- $\beta$ (TGF- $\beta$ ) receptor I; DAPI, 4',6-diamidino-2-phenylindole; WT, wild type.

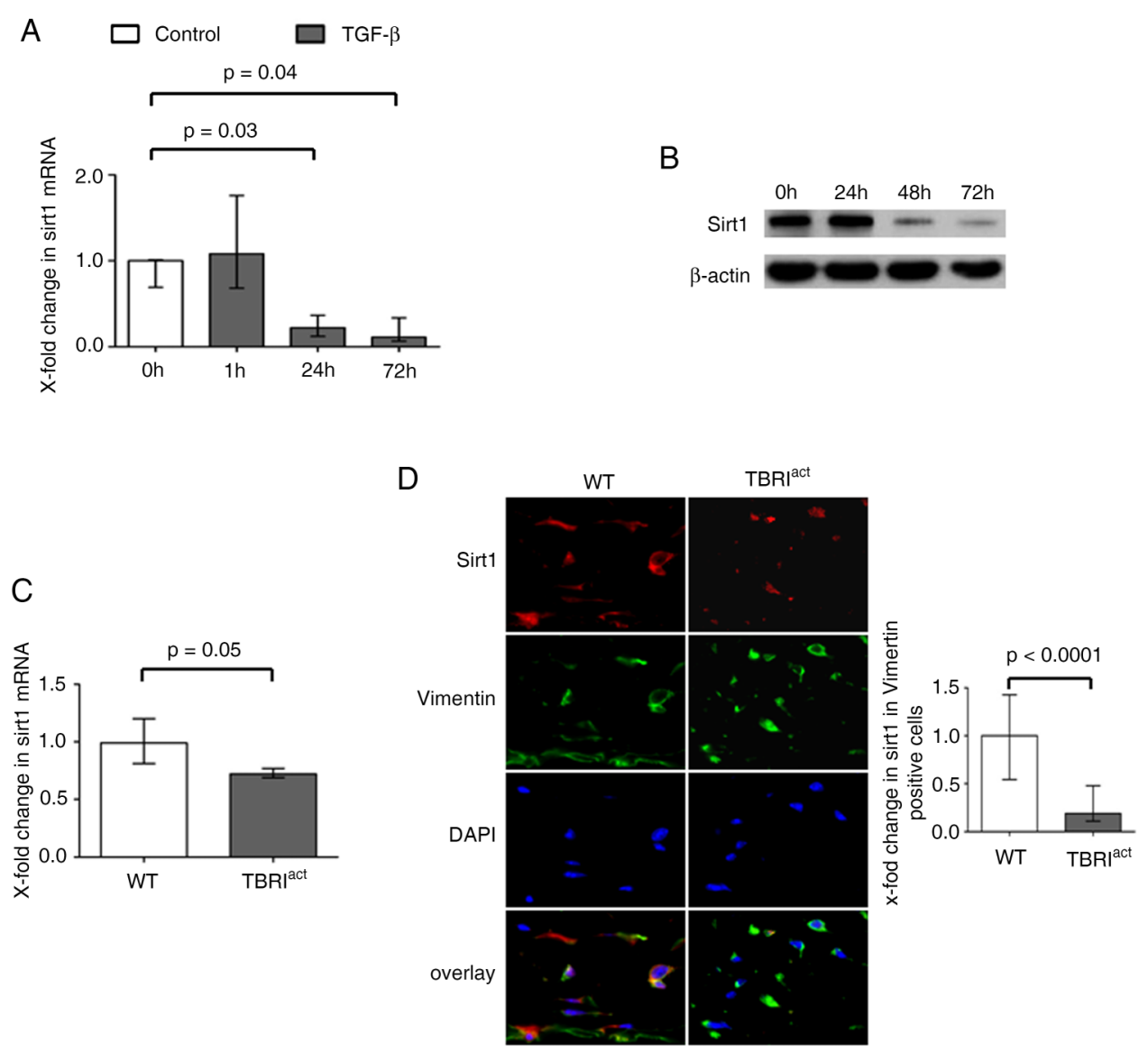

A

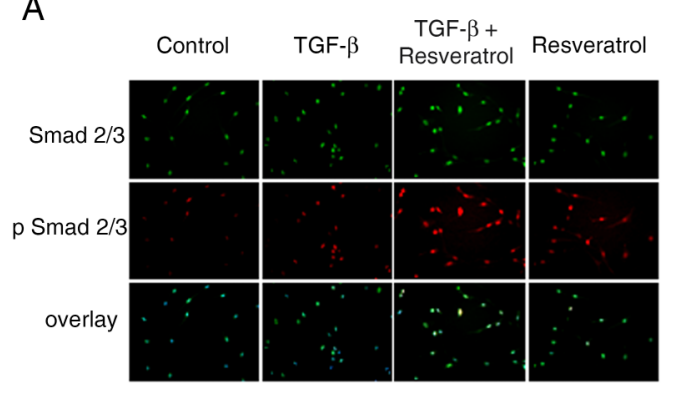

D

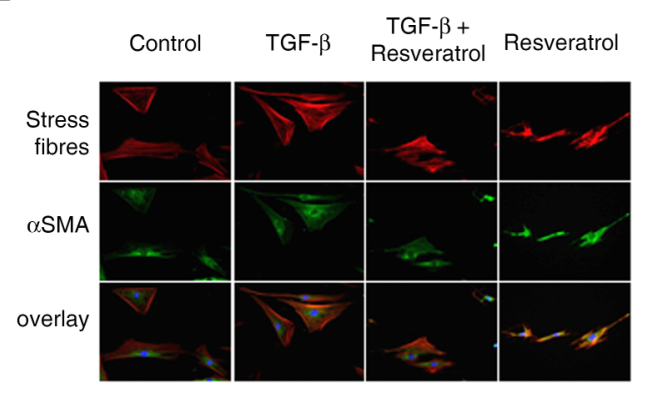

B

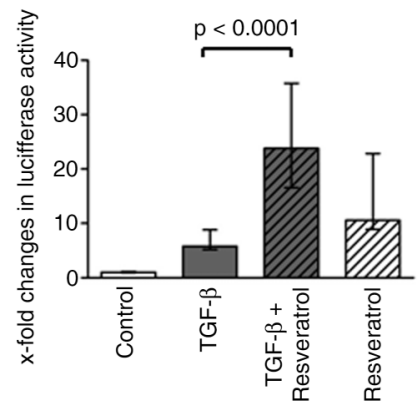

E

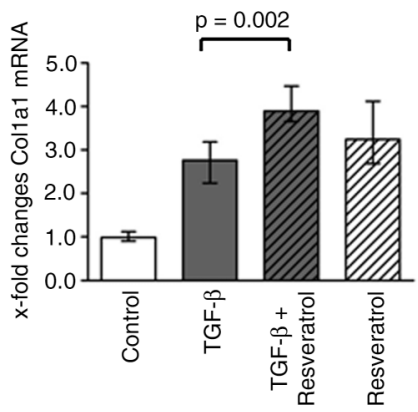

C

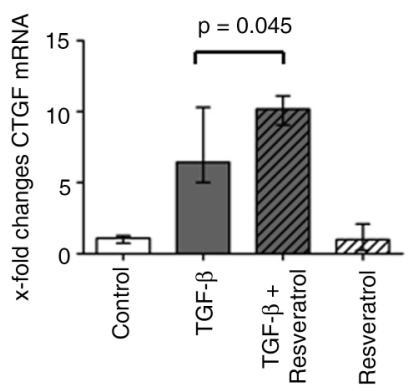

F

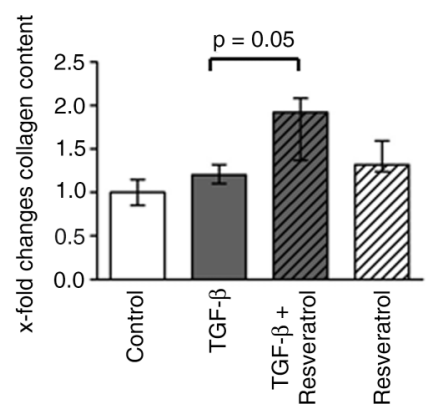

Figure 3 Effects of Sirt1 activation on transforming growth factor $\beta$ (TGF- $\beta$ ) signalling in cultured fibroblasts. Levels of total Smad3 and phosphorylated Smad2 and 3 (pSmad2/3) upon incubation with TGF- $\beta$, resveratrol and TGF- $\beta$ plus resveratrol shown by immunofluorescence (A). Smad/CAGA reporter activity (B), connective tissue growth factor (CTGF) mRNA (C), formation of stress fibres and $\alpha$-smooth muscle actin ( $\alpha$ SMA) protein expression levels (D), Col1a1 mRNA (E) and collagen release (F) in culture upon TGF- $\beta$ and resveratrol stimulation ( $\mathrm{n} \geq 8$ for each experimental condition and each outcome). Representative images are shown at a 200-fold magnification. All data are presented as median with IQR. 
resveratrol did not only enhance the TGF- $\beta$-induced accumulation of phosphorylated Smad 2 and $3(\mathrm{pSmad} 2 / 3)$, but was sufficient to induce accumulation of $\mathrm{pSmad} 2 / 3$ on its own (figure $3 \mathrm{~A}$ ). Resveratrol also stimulated the activity in Smad/CAGA reporter (figure $3 \mathrm{~B}$ ) and upregulated the mRNA levels of connective tissue growth factor, a classical TGF- $\beta / S$ mad target gene in dermal fibroblasts (figure 3C).

Sirt 1 activation also enhanced the profibrotic effects of TGF- $\beta$ and fostered the TGF- $\beta$-induced differentiation of resting fibroblasts into myofibroblasts with increased expression of $\alpha \mathrm{SMA}$ and formation of stress fibres (figure 3D). The Sirt1 agonist resveratrol also amplified the stimulatory effects of TGF- $\beta$ on the mRNA levels of col1a1 and on collagen protein released into the supernatant (figures 3E, F). Moreover, incubation with resveratrol also modestly increased myofibroblast differentiation and collagen release from human dermal fibroblasts in the absence of recombinant TGF- $\beta$ (figures 3D, F). Together, these data demonstrate that Sirt1 activation by resveratrol stimulates TGF- $\beta$ signalling and resident fibroblasts to release collagen.

\section{Fibroblast-specific knockdown of Sirt1 ameliorates experimental fibrosis}

We next investigated whether knockdown of Sirt1 can prevent experimental fibrosis. We selectively inactivated Sirt1 in fibroblasts using Sirt $1^{\mathrm{f} / \mathrm{fl}}$ mice and Col1a2; CreER mice. Sirt $1^{\mathrm{fl} / \mathrm{fl}}$; Fib
Cre ON mice lacking Sirt1 expression in fibroblasts did not display a basal skin phenotype and demonstrated normal skin histology and collagen content of the skin (figure 4A-D).

We first examined the outcome of fibroblast-specific knockdown of Sirt1 in the mouse model of bleomycin-induced skin fibrosis. ${ }^{25}$ Sirt $1^{\mathrm{f} / \mathrm{fl}}$; Fib Cre ON mice were partially protected from bleomycin-induced fibrosis (figure 4A). Bleomycin-induced dermal thickening, differentiation of resting fibroblasts into myofibroblasts and hydroxyproline accumulation were significantly reduced in bleomycin-challenged $\operatorname{Sirt}^{\mathrm{fl} / \mathrm{fl}}$; Fib Cre ON mice compared with Sirt $1^{\mathrm{fl} / \mathrm{fl}}$; Fib Cre OFF littermates (figure 4B-D). Consistent with the results in cultured fibroblasts, knockout of Sirt1 decreased the levels of $\mathrm{pSmad} 2 / 3$ in bleomycin-induced skin fibrosis (figure 4E).

Fibroblast-specific knockout of Sirt1 also ameliorated TBRIact-induced fibrosis. Sirt $1^{\mathrm{fl} / \mathrm{fl}}$; Fib Cre ON mice demonstrated reduced TBRIact-induced fibrosis (figure 5A) with decreased skin thickening, reduced myofibroblast counts and a lower hydroxyproline content compared with Sirt $1^{\mathrm{fl} / \mathrm{fl}}$; Fib Cre OFF mice (Figure 5B-D) and reduced the levels of $\mathrm{pSmad} 2 / 3$ (figure 5E).

Together, these data demonstrate that effective inactivation of Sirt 1 in fibroblasts exerts potent antifibrotic effects in murine models of early inflammatory as well as of later, noninflammatory stages of SSc.
A

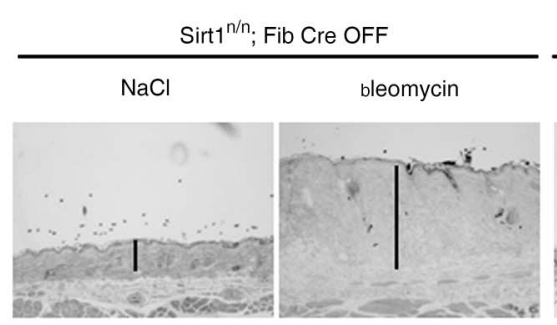

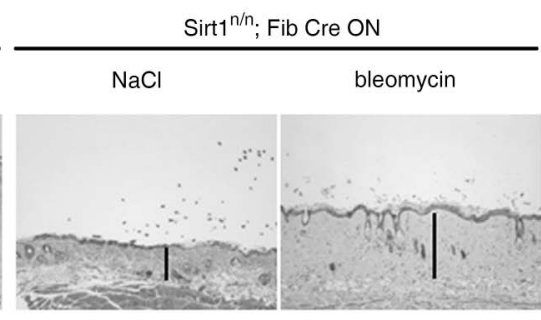

B

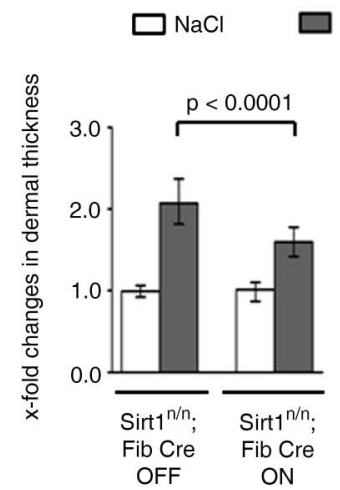

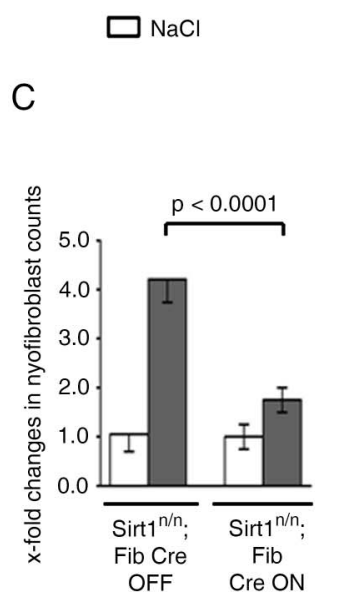
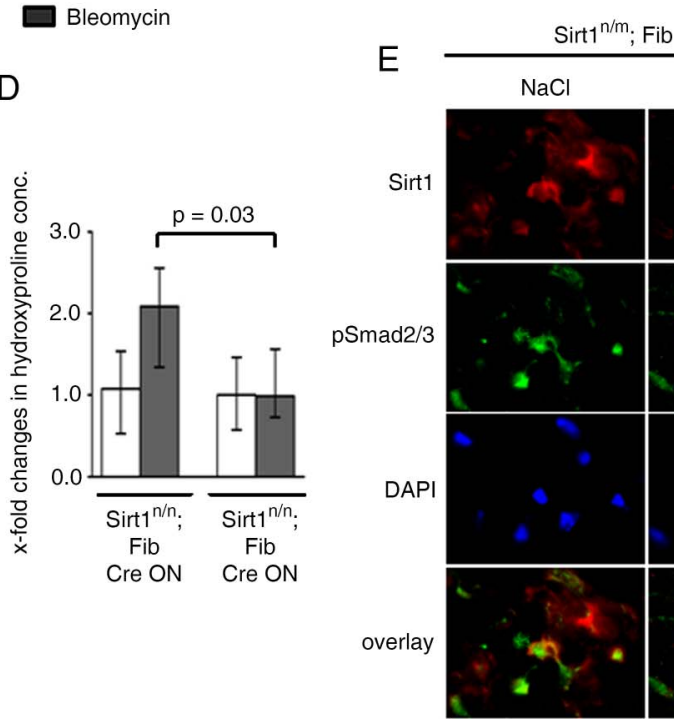

Fib Cre OFF

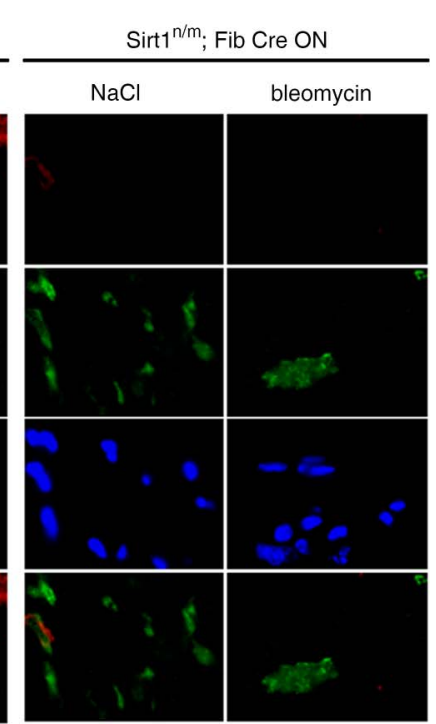

Figure 4 Fibroblast-specific knockout of Sirt1 ameliorates bleomycin-induced fibrosis. Histological features (A), dermal thickness (B), myofibroblast counts (C) and hydroxyproline content (D) upon challenge with bleomycin in mice with fibroblast-specific knockout of Sirt1 and control mice. Representative histological sections are shown at a 100-fold magnification. Vertical black bars indicate the dermal thickness. (E) Stainings for $\mathrm{pSmad} 2 / 3$ presented in 1000 -fold magnification. $\mathrm{n}=8$ for all groups of mice and all readouts. All data are presented as median with IQR. DAPI, 4',6-diamidino-2-phenylindole. 
A

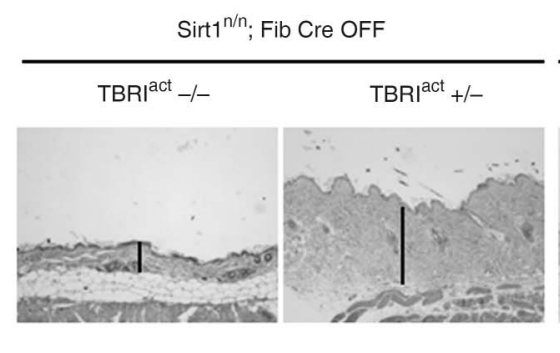

$\mathrm{TBRI}^{\mathrm{act}}-1-$

$\square$ TBRI $^{\text {act }}+/-$

C

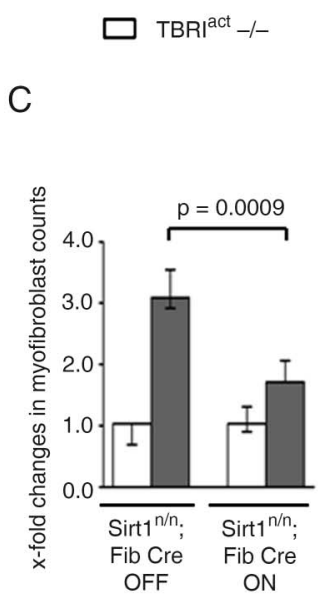

D

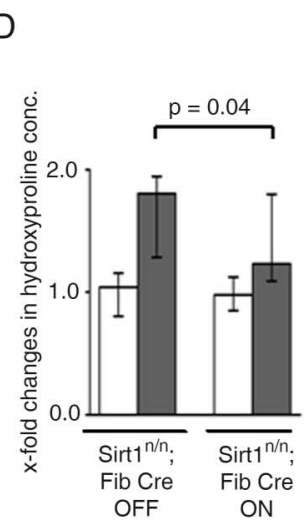

$\operatorname{Sirt1}^{\mathrm{n} / \mathrm{n}} ;$ Fib Cre ON

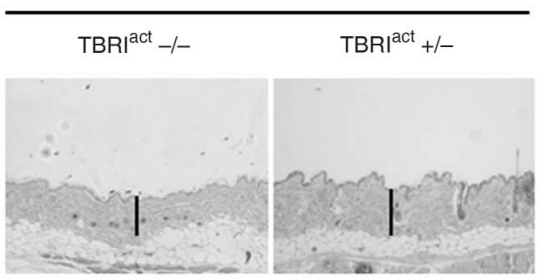

B

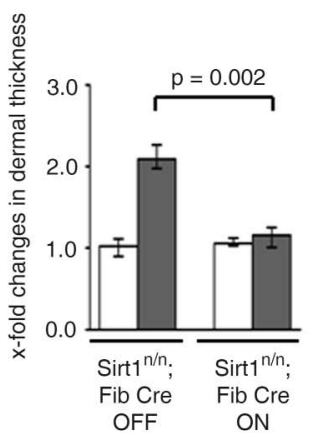

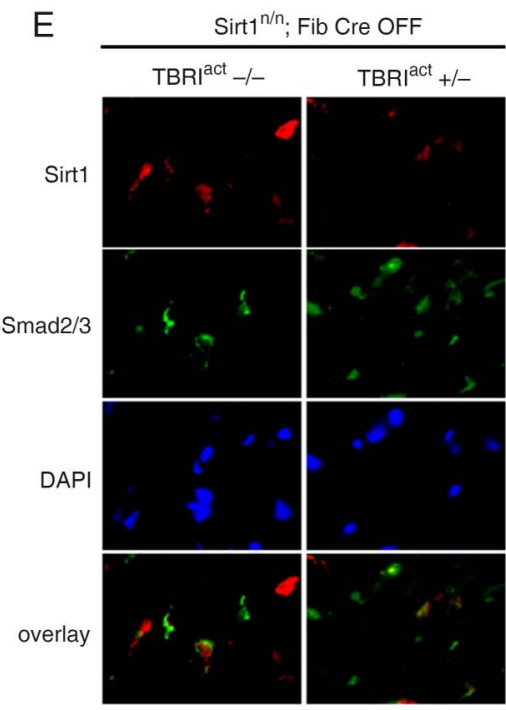

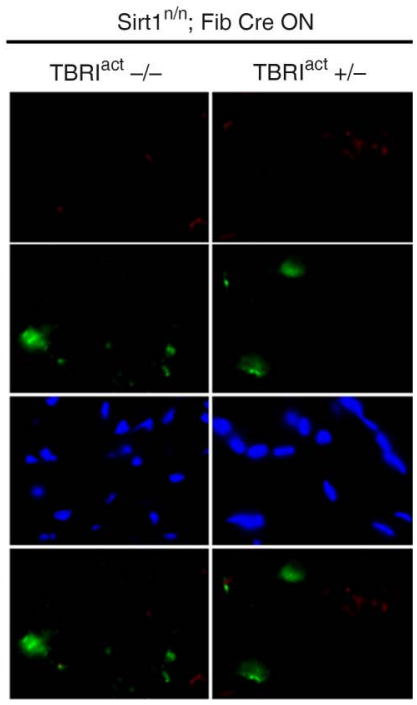

Figure 5 Fibroblast-specific knockout of Sirt1 reduces the profibrotic effects of TBRlact. Histological features (A), dermal thickness (B), myofibroblast counts $(C)$ and hydroxyproline content $(D)$ in mice with fibroblast-specific knockout of Sirt1 and control mice upon overexpression of TBRlact. Representative histological sections are shown at a 100-fold magnification. Vertical black bars indicate the dermal thickness. (E) Stainings for $\mathrm{pSmad} 2 / 3$ presented in 1000-fold magnification. $\mathrm{n}=8$ for all groups of mice and all readouts. All data are presented as median with IQR. TBRlact, active transforming growth factor- $\beta$ (TGF- $\beta$ ) receptor I; DAPI, 4',6-diamidino-2-phenylindole.

\section{DISCUSSION}

We demonstrate in the present study that Sirt 1 expression is decreased in fibroblasts in SSc skin and in murine models of skin fibrosis. Our data present first evidence that enhanced TGF- $\beta$ signalling mediates the downregulation of Sirt 1 in skin fibrosis: incubation with recombinant TGF- $\beta$ decreased the mRNA and protein levels of Sirt1 in cultured fibroblasts. Moreover, the expression of Sirt1 is decreased upon overexpression of a constitutively active TGF- $\beta$ receptor type $I$ in murine skin. These findings identify TGF- $\beta$ as a regulator of Sirt1 expression in SSc skin. However, although TGF- $\beta$ plays a central role, other differentially activated pathways in fibrosis such as impaired TNF- $\alpha$ signalling and oxidative stress may also influence the expression of Sirt1 in SSc. ${ }^{41}$

Furthermore, we show that Sirt1 positively regulates TGF- $\beta$ signalling in SSc fibroblasts. Activation of Sirt1 in vitro by treatment with resveratrol enhances the profibrotic effects of TGF- $\beta$ with increased Smad reporter activity, elevated expression of TGF- $\beta$ target genes, increased differentiation of resting fibroblasts into myofibroblasts and enhanced collagen release. However, the studies with resveratrol need further confirmation: although resveratrol has been used as a bona fide activator of Sirt1 in numerous studies (reviewed in $^{7}$ ), recent studies question the selectivity of Sirt1 with additional effects on other signalling cascades such as nuclear factor- $\mathrm{B}$ and canonical Wnt signalling, and challenge the ability of Sirt1 to activate the catalytic activity of Sirt $1 .{ }^{42} 43$ Moreover, the effects of resveratrol are highly dose dependent. This is exemplified by its effects on canonical Wnt signalling, which is inhibited by low doses, but activated by higher doses of resveratrol. ${ }^{44}{ }^{45}$ We thus confirm our results using a genetic strategy. We demonstrate in vivo using Cre-Lox mediated, fibroblast-specific knockdown of Sirt1 strongly ameliorates the stimulatory effects of TGF- $\beta$ on fibroblasts and reduces the expression of TGF- $\beta$ target genes, impairs myofibroblast differentiation and reduces the release of collagen. Sirt1 may modulate several targets associated with TGF- $\beta$ signalling to achieve its potent modulatory effects: Sirt1 negatively regulates the expression of Smad7, an endogenous inhibitor of canonical Smad signalling in fibroblasts ${ }^{8}$ and may thereby promote TGF- $\beta /$ Smad-dependent transcription. Moreover, Sirt1 represses the expression of peroxisome proliferator-activated receptor $\gamma$, which has been characterised as an important inhibitor of TGF- $\beta$ signalling. ${ }^{46}$ Furthermore, Sirt1 stimulates the expression of the transcription factor $\mathrm{Nrf} 2,{ }^{47}$ which has been shown to mediate the deleterious effects of ROS in experimental skin fibrosis (Varga J, unpublished data). Finally, activation of Sirt1 renders fibroblasts more resistant to fibrosis and may thereby interfere with termination of fibroblast activation. ${ }^{4148}$ 
The stimulatory effects of Sirt 1 on TGF- $\beta$ signalling together with the inhibitory effects of TGF- $\beta$ on Sirt 1 expression suggest that the downregulation of Sirt1 by TGF- $\beta$ serves as an endogenous negative feedback loop to limit TGF- $\beta$ signalling in fibroblasts. However, the moderate decrease of Sirt 1 in SSc skin is insufficient to counterbalance the extensive and persistent activation of TGF- $\beta$ signalling in SSc fibroblasts. Nevertheless, our experiments with cultured fibroblasts stimulated with resveratrol and with mice lacking Sirt1 in fibroblasts demonstrate that augmentation of this endogenous feedback loop by targeting Sirt 1 potently inhibits TGF- $\beta$ signalling and ameliorates fibrosis. Thus, inhibitors of Sirt1, which are currently in clinical development, may be a novel approach to inhibit TGF- $\beta$ signalling in fibrotic diseases.

Careful further analyses of the role of Sirt1 in different cell types, different organ systems and additional models are warranted as the effect of Sirt1 differs depending on the target cell and organ, as well as on the activation level. We demonstrate in the present study that inactivation of Sirt1 in fibroblasts exerts an antifibrotic effect and that fibroblast-specific knockdown of Sirt1 strongly ameliorates fibrosis in bleomycin-induced and TBRIactinduced skin fibrosis, two well-established models of skin fibrosis with distinct underlying pathomechansims. In contrast, targeting Sirt1 in epithelial cells in experimental renal fibrosis results in opposite effects with exacerbation of experimental kidney fibrosis. ${ }^{49}$ Thus, while knockdown of Sirt1 prevents the activation of resident fibroblasts, it also promotes epithelial-to-mesenchymaltransition (EMT) in epithelial cells in the kidney. ${ }^{49} 50$ Together, these data suggest that Sirt1 may have profibrotic effects in organ systems, in which fibrosis is mainly driven by activation of resident fibroblasts as in skin, whereas it prevents EMT to limit fibrosis in epithelial organs such as the kidney. Thus, additional studies with cell-type-specific inactivation of Sirt 1 in different epithelial and mesenchymal cell types and in different organs are required to further elucidate the role of Sirt1 in fibrosis. Considering the potential role of Sirt1 in response to oxidative stress, it would also be interesting to study the effect of Sirt1 inactivation in further models of fibrosis depending on oxidative stress such as in $\mathrm{HOCl}$-induced fibrosis. Moreover, preclinical models with vascular manifestations such as Fra2 transgenic mice or mice deficient in urokinase-type plasminogen activator receptor would provide further important information.

Our data identify Sirt1 as an endogenous amplifier of TGF- $\beta$ signalling and fibrosis: patients with SSc try to counteract the hyperactivation of TGF- $\beta$ signalling by decreasing Sirt 1 expression. However, the modest downregulation of Sirt1 is not sufficient to inhibit the excessive activation of fibroblasts in SSc. We demonstrate with genetic and pharmacological approaches that amplification of this endogenous regulatory loop by genetic inactivation or pharmacological inhibition exerts potent antifibrotic effects. These findings provide a novel concept for the treatment of fibrosis: rather than trying to 'correct' a pathological hyperactivation or hypoactivation of a given pathway, inhibition of Sirt1 further promotes an endogenous, partially active counter regulatory mechanism. Although several inhibitors of Sirt1 are in clinical development, the cell-type-specific and tissue-specific effects of Sirt1 demand further studies and may argue against Sirt1 as a therapeutic target to date.

Acknowledgements We thank Katja Dreißigacker, Regina Kleinlein, Rosella Mancuso and Verena Wäsch for excellent technical assistance.

Contributors Design of the study: PZ and JHWD. Acquisition of data: PZ, KP-Z, $J H, M T$ and BS. Interpretation of data: PZ, KP-Z, JH, MT, BS, OD, GS and JHWD. Manuscript preparation: PZ and JHWD.
Funding Grants DI 1537/5-1, DI 1537/7-1, DI 1537/8-1, DI 1537/9-1, and SCHE 1583/7-1 of the Deutsche Forschungsgesellschaft, grants A57 of the IZKF in Erlangen, the ELAN-Program of the University of Erlangen-Nuremberg and the Career Support Award of Medicine of the Ernst Jung Foundation. Supported by CMH Research Project 0000023728.

Competing interests OD has consultancy relationships and/or has received research funding from Actelion, Pfizer, Ergonex, BMS, Sanofi-Aventis, United BioSource Corporation, medac, Biovitrium, Boehringer Ingelheim, Novartis, 4D Science and Active Biotech in the area of potential treatments of scleroderma; JHWD has consultancy relationships and/or has received research funding from Actelion, Pfizer, Ergonex, BMS, Celgene, Bayer Pharma, Boehringer Ingelheim, JB

Therapeutics, Sanofi-Aventis, Novartis, Array Biopharma, KaroBio and Active Biotech in the area of potential treatments of scleroderma and is stock owner of 4D Science.

\section{Patient consent Obtained.}

Ethics approval University of Erlangen-Nuremberg.

Provenance and peer review Not commissioned; externally peer reviewed.

\section{REFERENCES}

1 Gabrielli A, Avvedimento EV, Krieg T. Scleroderma. N Engl J Med 2009;360:1989-2003

2 Tyndall AJ, Bannert B, Vonk $M$, et al. Causes and risk factors for death in systemic sclerosis: a study from the EULAR Scleroderma Trials and Research (EUSTAR) database. Ann Rheum Dis 2010;69:1809-15.

3 Samarakoon R, Overstreet JM, Higgins PJ. TGF-beta signaling in tissue fibrosis: redox controls, target genes and therapeutic opportunities. Cell Signal 2013;25:264-8

4 Houtkooper RH, Pirinen E, Auwerx J. Sirtuins as regulators of metabolism and healthspan. Nat Rev Mol Cell Biol 2012;13:225-38.

5 Lavu S, Boss O, Elliott PJ, et al. Sirtuins--novel therapeutic targets to treat age-associated diseases. Nat Rev Drug Discov 2008;7:841-53.

6 Blander G, Guarente L. The Sir2 family of protein deacetylases. Annu Rev Biochem 2004;73:417-35.

7 Pillarisetti S. A review of Sirt1 and Sirt1 modulators in cardiovascular and metabolic diseases. Recent Pat Cardiovasc Drug Discov 2008;3:156-64.

8 Kume S, Haneda M, Kanasaki K, et al. SIRT1 inhibits transforming growth factor beta-induced apoptosis in glomerular mesangial cells via Smad7 deacetylation. J Biol Chem 2007;282:151-8.

9 Li J, Qu X, Ricardo SD, et al. Resveratrol inhibits renal fibrosis in the obstructed kidney: potential role in deacetylation of Smad3. Am J Pathol 2010;177: 1065-71.

10 Yao H, Sundar IK, Ahmad T, et al. SIRT1 protects against cigarette smoke-induced lung oxidative stress via a FOXO3-dependent mechanism. Am J Physiol Lung Cell Mol Physiol 2014;306:L816-828.

11 Hasegawa K, Wakino S, Yoshioka K, et al. Sirt1 protects against oxidative stress-induced renal tubular cell apoptosis by the bidirectional regulation of catalase expression. BiochemBiophys Res Commun 2008;372:51-6.

12 Salminen A, Kaarniranta K, Kauppinen A. Crosstalk between Oxidative Stress and SIRT1: impact on the aging process. Int J Mol Sci 2013;4:3834-3859.

13 Ohguchi K, Itoh T, Akao Y, et al. SIRT1 modulates expression of matrix metalloproteinases in human dermal fibroblasts. Br J Dermatol 2010;163: 689-94.

14 van den Hoogen F, Khanna D, Fransen J, et al. 2013 classification criteria for systemic sclerosis: an American college of rheumatology/European league against rheumatism collaborative initiative. Ann Rheumat Dis 2013;72:1747-55.

15 Akhmetshina A, Palumbo K, Dees C, et al. Activation of canonical Wnt signalling is required for TGF-beta-mediated fibrosis. Nat Commun 2012;3:735.

16 Dees C, Tomcik M, Palumbo-Zerr K, et al. JAK-2 as a novel mediator of the profibrotic effects of transforming growth factor beta in systemic sclerosis. Arthritis Rheum 2012;64:3006-15.

17 Dees C, Tomcik M, Zerr $\mathrm{P}$, et al. Notch signalling regulates fibroblast activation and collagen release in systemic sclerosis. Ann Rheum Dis. 2011;70:1304-10.

18 Horn A, Palumbo K, Cordazzo C, et al. Hedgehog signaling controls fibroblast activation and tissue fibrosis in systemic sclerosis. Arthritis Rheum 2012;64:2724-33.

19 Reich N, Maurer B, Akhmetshina A, et al. The transcription factor Fra-2 regulates the production of extracellular matrix in systemic sclerosis. Arthritis Rheum 2010;62:280-90.

20 Palumbo K, Zerr P, Tomcik M, et al. The transcription factor JunD mediates transforming growth factor \{beta\}-induced fibroblast activation and fibrosis in systemic sclerosis. Ann Rheum Dis 2011;70:1320-6.

21 Reich $\mathrm{N}$, Tomcik M, Zerr $\mathrm{P}$, et al. Jun N-terminal kinase as a potential molecular target for prevention and treatment of dermal fibrosis. Ann Rheum Dis 2012;71:737-45.

22 Dees $C$, Akhmetshina $A$, Zerr $P$, et al. Platelet-derived serotonin links vascular disease and tissue fibrosis. J Exp Med 2011;208:961-72. 
23 Dees C, Schlottmann I, Funke R, et al. The Wnt antagonists DKK1 and SFRP1 are downregulated by promoter hypermethylation in systemic sclerosis. Ann Rheum Dis 2014;73:1232-9.

24 Beyer C, Zenzmaier C, Palumbo-Zerr K, et al. Stimulation of the soluble guanylate cyclase (sGC) inhibits fibrosis by blocking non-canonical TGFbeta signalling. Ann Rheum Dis 2015;74:1408-16.

25 Beyer C, Schett G, Distler 0, et al. Animal models of systemic sclerosis: prospects and limitations. Arthritis Rheum 2010;62:2831-44.

26 Distler A, Lang V, Del Vecchio T, et al. Combined inhibition of morphogen pathways demonstrates additive antifibrotic effects and improved tolerability. Ann Rheum Dis 2014;73:1264-8

27 Distler A, Ziemer C, Beyer C, et al. Inactivation of evenness interrupted (EVI) reduces experimental fibrosis by combined inhibition of canonical and non-canonical Wnt signalling. Ann Rheum Dis 2014;73:624-7.

28 Distler JH, Jungel A, Huber LC, et al. Imatinib mesylate reduces production of extracellular matrix and prevents development of experimental dermal fibrosis. Arthritis Rheum 2007;56:311-22.

29 Sonnylal S, Denton CP, Zheng B, et al. Postnatal induction of transforming growth factor beta signaling in fibroblasts of mice recapitulates clinical, histologic, and biochemical features of scleroderma. Arthritis Rheum 2007;56:334-44.

30 Dees C, Zerr P, Tomcik M, et al. Inhibition of Notch signaling prevents experimental fibrosis and induces regression of established fibrosis. Arthritis Rheum 2011;63:1396-404.

31 Horn A, Kireva T, Palumbo-Zerr K, et al. Inhibition of hedgehog signalling prevents experimental fibrosis and induces regression of established fibrosis. Ann Rheum Dis 2012;71:785-9.

32 Weingartner $S$, Zerr $P$, Tomcik $M$, et al. Pomalidomide is effective for prevention and treatment of experimental skin fibrosis. Ann Rheum Dis. 2012;71:1895-9.

33 Beyer C, Reich N, Schindler SC, et al. Stimulation of soluble guanylate cyclase reduces experimental dermal fibrosis. Ann Rheum Dis 2012;71:1019-26.

34 Palumbo-Zerr K, Horn A, Distler A, et al. Inactivation of fatty acid amide hydrolase exacerbates experimental fibrosis by enhanced endocannabinoid-mediated activation of CB1. Ann Rheum Dis 2012;71:2051-4.

35 Tomcik M, Zerr P, Pitkowski J, et al. Heat shock protein 90 (Hsp90) inhibition targets canonical TGF-beta signalling to prevent fibrosis. Ann Rheum Dis 2014;73:1215-22

36 Zhang Y, Dees C, Beyer C, et al. Inhibition of casein kinase II reduces TGFbeta induced fibroblast activation and ameliorates experimental fibrosis. Ann Rheum Dis 2015;74:936-43.
37 Akhmetshina A, Dees C, Busch N, et al. The cannabinoid receptor CB2 exerts antifibrotic effects in experimental dermal fibrosis. Arthritis Rheum 2009;60:1129-36.

38 Avouac J, Palumbo K, Tomcik M, et al. Inhibition of activator protein 1 signaling abrogates transforming growth factor beta-mediated activation of fibroblasts and prevents experimental fibrosis. Arthritis Rheum 2012;64:1642-52.

39 Beyer $C$, Huang J, Beer J, et al. Activation of liver $X$ receptors inhibits experimental fibrosis by interfering with interleukin- 6 release from macrophages. Ann Rheum Dis 2015;74:1317-24.

40 Beyer $\mathrm{C}$, Reichert $\mathrm{H}$, Akan $\mathrm{H}$, et al. Blockade of canonical Wnt signalling ameliorates experimental dermal fibrosis. Ann Rheum Dis 2013;72:1255-8.

41 Niederer F, Ospelt C, Brentano F, et al. SIRT1 overexpression in the rheumatoid arthritis synovium contributes to proinflammatory cytokine production and apoptosis resistance. Ann Rheum Dis. 2011;70:1866-73.

42 Beher D, Wu J, Cumine $\mathrm{S}$, et al. Resveratrol is not a direct activator of SIRT1 enzyme activity. Chem Biol Drug Des 2009;74:619-24.

43 Pacholec M, Bleasdale JE, Chrunyk B, et al. SRT1720, SRT2183, SRT1460, and resveratrol are not direct activators of SIRT1. J Biol Chem 2010;285: 8340-51.

44 Hope C, Planutis K, Planutiene M, et al. Low concentrations of resveratrol inhibit Wnt signal throughput in colon-derived cells: implications for colon cancer prevention. Mol Nutr Food Res 2008;52(Suppl 1):S52-61.

45 Zhou H, Shang L, Li X, et al. Resveratrol augments the canonical Wnt signaling pathway in promoting osteoblastic differentiation of multipotent mesenchymal cells. Exp Cell Res 2009;315:2953-62.

46 Wei J, Bhattacharyya S, Varga J. Peroxisome proliferator-activated receptor gamma: innate protection from excessive fibrogenesis and potential therapeutic target in systemic sclerosis. Curr Opin Rheumatol 2010;22:671-6.

47 Huang $\mathrm{K}$, Huang J, Xie $\mathrm{X}$, et al. Sirt1 resists advanced glycation end products-induced expressions of fibronectin and TGF-beta1 by activating the Nrf2/ ARE pathway in glomerular mesangial cells. Free Radic Biol Med 2013;65:528-40.

48 Wang W, Yan C, Zhang J, et al. SIRT1 inhibits TNF-alpha-induced apoptosis of vascular adventitial fibroblasts partly through the deacetylation of Fox01. Apoptosis 2013;18:689-701.

49 Simic P, Williams EO, Bell EL, et al. SIRT1 suppresses the epithelial-to-mesenchymal transition in cancer metastasis and organ fibrosis. Cell Rep 2013;3:1175-86.

50 Huang $X$, Wen $D$, Zhang $M$, et al. Sirt1 activation ameliorates renal fibrosis by inhibiting the TGF-beta/Smad3 Pathway. J Cell Biochem 2014;115:996-1005. 


\section{ARD Sirt1 regulates canonical TGF- $\beta$ signalling to control fibroblast activation and tissue fibrosis}

Pawel Zerr, Katrin Palumbo-Zerr, Jingang Huang, Michal Tomcik,

Barbora Sumova, Oliver Distler, Georg Schett and Jörg H W Distler

Ann Rheum Dis2016 75: 226-233 originally published online September 1,2014

doi: 10.1136/annrheumdis-2014-205740

Updated information and services can be found at:

http://ard.bmj.com/content/75/1/226

These include:

Supplementary Supplementary material can be found at:

Material http://ard.bmj.com/content/suppl/2014/08/22/annrheumdis-2014-2057 40.DC1

References This article cites 50 articles, 21 of which you can access for free at: http://ard.bmj.com/content/75/1/226\#ref-list-1

Email alerting Receive free email alerts when new articles cite this article. Sign up in the service box at the top right corner of the online article.

Topic

Articles on similar topics can be found in the following collections

Connective tissue disease (4253)

Immunology (including allergy) (5144)

\section{Notes}

To request permissions go to:

http://group.bmj.com/group/rights-licensing/permissions

To order reprints go to:

http://journals.bmj.com/cgi/reprintform

To subscribe to BMJ go to:

http://group.bmj.com/subscribe/ 